\title{
Multiple sclerosis: High prevalence of the 'central vein' sign in white matter lesions on susceptibility-weighted images
}

The Neuroradiology Journal 0(00) 1-6

(C) The Author(s) 2018

Reprints and permissions: sagepub.co.uk/journalsPermissions.nav DOI: 10.1177/1971400918763577 journals.sagepub.com/home/neu @SAGE

\author{
Gianvincenzo Sparacia ${ }^{1}$ (D), Francesco Agnello ${ }^{1}$, Angelo Gambino ${ }^{1}$, \\ Martina Sciortino ${ }^{2}$ and Massimo Midiri $^{1}$
}

\begin{abstract}
Purpose: The aim of this study was to determine the occurrence and distribution of the 'central vein' sign in white matter lesions on susceptibility-weighted magnetic resonance images in patients with multiple sclerosis (MS) and cerebral small vessel disease (CSVD).

Materials and methods: T2-weighted and fluid-attenuated inversion recovery magnetic resonance images of 19 MS patients and 19 patients affected by CSVD were analysed for the presence and localisation of focal hyperintense white matter lesions. Lesions were subdivided into periventricular or non-periventricular (juxtacortical, subcortical, deep white matter and cerebellar) distributed. The number and localisation of lesions presenting with the central vein sign were recorded and compared between MS and CSVD lesions.

Results: A total of 313 MS patients and 75 CSVD lesions were identified on T2-weighted and fluid-attenuated inversion recovery magnetic resonance images. The central vein sign was found in 128 MS lesions (40.9\%), and the majority of them $(71 / 128,55.5 \%)$ had a periventricular distribution. The central vein sign was found in 22 out of 75 (29.3\%) CSVD lesions, and periventricular distribution was seen in six out of 22 (27.2\%) CSVD lesions. The difference in the proportion of white matter hyperintense lesions that presented with the central vein sign on susceptibility-weighted images in patients with MS and CSVD was statistically different, and a significantly higher number of MS patients presented with lesions with the central vein sign compared to CSVD patients.

Conclusion: The presence of the central vein sign on susceptibility-weighted images for MS lesions improves the understanding of the periventricular distribution of MS lesions and could contribute as adjunctive diagnostic criteria for MS disease.
\end{abstract}

\section{Keywords}

Central vein sign, susceptibility-weighted imaging, multiple sclerosis, cerebral small vessel disease, magnetic resonance imaging

\section{Introduction}

Multiple sclerosis (MS) is the most common autoimmune neurological disorder affecting young adults. It is pathologically characterised by multifocal inflammation, demyelination, axonal injury and neuronal loss. ${ }^{1}$

Conventional magnetic resonance imaging (MRI) has high sensitivity in demonstrating MS dissemination in time and space. ${ }^{1,2}$ The current diagnosis of MS is based on a combination of clinical, radiological and laboratory findings. ${ }^{2}$ However, MRI specificity for the diagnosis of MS is relatively low because several vascular, infectious, neoplastic, congenital and metabolic diseases can show a similar radiological appearance and mimic MS at MRI. ${ }^{3,4}$ MS is thus usually a diagnosis of exclusion.
Efforts have been made to improve the ability of imaging to differentiate MS from its mimic. One option may be to detect a small central vein (the 'central vein' sign) in MS lesions. Indeed, pathological studies at autopsy have demonstrated that the typical extension and morphology of the MS lesions is due to the perivenous localisation in the white matter of the brain and spine. ${ }^{5,6}$ As a result of high sensitivity to slow venous flow, the utility of susceptibility-weighted

${ }^{1}$ DIBIMED - Sezione di Scienze Radiologiche, University of Palermo, Palermo, Italy

${ }^{2}$ School of Medicine, University of Palermo, Palermo, Italy

\section{Corresponding author:}

Gianvincenzo Sparacia, DIBIMEF - Sezione di Scienze Radiologiche, University of Palermo, Via Del Vespro, 127, 90127 Palermo, Italy. Email: gianvincenzo.sparacia@unipa.it 
imaging (SWI) sequence has recently been investigated for the detection of the central vein sign. ${ }^{7,8}$

In a small prospective study, Kau et al. ${ }^{7}$ have reported that although the central vein sign was not exclusively present in MS lesions it helps differentiate MS from non-MS lesions. More recent studies at ultrahigh-field have demonstrated that the presence of a central vein improves the differentiation of MS and vascular lesions. ${ }^{9-11}$ In addition, Lane et al. ${ }^{8}$ have reported that the detection of the central vein sign helps differentiate MS from dementia.

There is also an increasing acceptance of the potential role of the central vein sign as a biomarker of inflammatory demyelination in the most recent guidelines for MS diagnosis. ${ }^{12-15}$ However, further research is required to consider the central vein sign a reliable diagnostic criterion of MS. As the most common differential diagnosis for MS is focal white matter hyperintensity due to cerebral small vessel disease (CSVD), the purpose of our study was to compare the occurrence and distribution of the central vein sign in white matter lesions among patients with MS and CSVD.

\section{Materials and methods}

This retrospective study was approved by the institutional review board of our institution, and informed consent was waived; however, informed written consent for the MRI was obtained in all patients.

This study included 19 patients (nine men, 10 women; aged 19-53 years, mean age 36.9 years) with MS and 19 patients (nine men, 10 women; aged 59-72 years, mean age 61.2 years) with CSVD. All MS patients underwent brain MRI examination as part of the routine follow-up. MRI examinations were performed between December 2016 and April 2017.

Inclusion criteria for MS patients were age over 18 years, clinical diagnosis of MS and fulfillment of 2010 revised McDonald criteria for $\mathrm{MS}^{2}{ }^{2}$ The mean disease duration for MS was $8.1 \pm 6.5$ years. Eleven patients $(57.9 \%)$ had the relapsing-remitting clinical subtype, six $(31.5 \%)$ had the secondary progressive clinical subtype, and two $(10.5 \%)$ had the primary progressive clinical subtype. The Expanded Disability Status Scale (EDSS) of the patients with MS ranged between 1 and 4.5 , with a mean of $1.9 \pm 0.8$.

Diagnosis of CSVD required lack of symptoms suggestive of another white matter disease, identification of vascular risk factors, and the presence of cerebral white matter hyperintensities on T2-weighted and fluid-attenuated inversion recovery (FLAIR) MRI, as commonly seen in CSVD.

\section{MRI protocol}

All MRI examinations were performed on a 1.5 T MR scanner (Achieva, Philips Medical Systems, Best, The Netherlands) using an 8-channel phased-array head coil.
The MRI protocol included axial and sagittal fastspin echo T2-weighted sequences (TR/TE, 5100/110); axial FLAIR sequences (TR/TE/TI, 8000/140/2400); axial, sagittal and coronal contrast-enhanced $(0.1 \mathrm{mmol} / \mathrm{kg}$ of gadobutrol; Gadovist, Bayer, Bonn, Germany) fast-spin echo T1-weighted sequences (TR/TE, 650/12); field-of-view (FOV) $22 \mathrm{~cm}$; matrix $320 \times 320$; slice thickness $5 \mathrm{~mm}$; intersection gap $1 \mathrm{~mm}$; number of excitations 2 .

The SWI sequences were obtained using a technique that combines a long-TE high-resolution fully flow-compensated 3DGRE sequence with filtered phase information in each voxel both to enhance the contrast in magnitude images and add the susceptibility differences between tissues as a new source of information. ${ }^{15-18}$

The SWI images were obtained without the injection of contrast material (TR/TE, 34/24; flip angle $10^{\circ}$; FOV $22 \mathrm{~cm}$; matrix $256 \times 512$; slice thickness $1.2 \mathrm{~mm}$; no intersection gap; number of images 140; number of excitations 1; and acquisition time 5.40 minutes). The SWI images were also post-processed with the minimum intensity projection (MinIP) algorithm in the axial plane with a slice thickness of 3-10 $\mathrm{mm}$ to visualize better the 'signal void' of the vessel structures.

\section{Image evaluation}

The T2-weighted and FLAIR images, presented in random order on a picture archiving and communication system (Agfa HealthCare GmbH, Bonn, Germany), were analysed in consensus by two neuroradiologists, each with at least 10 years of experience who were unaware of the patients' clinical information, for the presence and localisation of focal hyperintense white matter lesions.

Lesions were subdivided into periventricular or nonperiventricular distributed. Non-periventricular lesions were subdivided into juxtacortical, subcortical, deep white matter and cerebellar. Only white matter lesions greater than $5 \mathrm{~mm}$ in largest diameter were considered, limiting the overall number of lesions greater than $3 \mathrm{~mm}$ in largest diameter to a maximum of eight. Confluent lesions were excluded. We chose these inclusion criteria that have been reported better to reflect equivocal cases in clinical routine and better reflect the critical lesion load regarding the Barkhof criteria. ${ }^{7}$

The SWI images were evaluated for the presence or absence of a venous structure within the MS lesions. To better differentiate the signal void of a vessel, the MinIP post-processed SWI images were also used. The presence of a vein within the MS lesion was defined according to the standard radiological definition established in the North American Imaging in Multiple Sclerosis (NAIMS) guidelines, ${ }^{15}$ which recommend that the vein appears as a thin hypointense line or small hypointense dot; when technically possible, the vein can be visualised in at least two perpendicular planes; the vein runs partially or entirely through the lesion, but must be located centrally regardless of the lesion's 
shape; the vein has a small $(<2 \mathrm{~mm})$ apparent diameter. As exclusion criteria, the standard radiological definition was also used: the lesion is less than $3 \mathrm{~mm}$ in diameter in any plane; the lesion merges with another lesion (confluent lesion); the lesion has multiple distinct veins; the lesion is poorly visible (as a result of motion or other MRI-related artifacts).

Lesions containing calcification, hemosiderin and iron accumulation, which mimic venous structures because they show a hypointense signal similar to venous structures, were excluded on the basis of the lack of signal void. Calcifications were excluded with the filtered phase images.

\section{Statistical analysis}

Statistical analysis was performed using a non-parametric Wilcoxon rank-sum test to compare the number of white matter lesions presenting with the central vein sign on SWI images in MS and CSVD patients, and the number of MS patients presenting with lesions with the central vein sign with the number of CSVD patients presenting with lesions with the central vein sign. Differences for a $P$ value of less than 0.05 were considered statistically significant. Data are presented as percentages and mean \pm standard deviations.

The statistical analysis was performed using SPSS Statistics software (SPSS, Chicago, IL, USA). As the sample size for this study was small, we assessed the power of the Wilcoxon rank-sum test with the analysis software G*Power (Heinrich,Heine,University, Düsseldorf, Germany) to ensure that a statistical power of 0.90 for the result was achieved.

\section{Results}

A total of $313 \mathrm{MS}$ lesions were identified in MS patients, while 75 cerebral white matter focal hyperintense lesions were identified in CSVD patients. All lesions were clearly visible both on the T2-weighted and FLAIR images.

In MS patients, periventricular distribution was found in 139 (44.4\%) lesions while non-periventricular distribution was found in $174(55.6 \%)$ lesions. The smallest MS lesion diameter was $3 \mathrm{~mm}$. A total of 128 MS lesions containing venous structures (the central vein sign) were detected out of 313 MS lesions $(40.9 \%)$, and the majority of them $(71 / 128,55.5 \%)$ had a periventricular distribution (Figures 1 and 2).

In CSVD patients, periventricular distribution was found in $31(41.3 \%)$ lesions while non-periventricular distribution was found in $44(58.7 \%)$ lesions. The central vein sign was found in 22 out of 75 (29.3\%) CSVD lesions and periventricular distribution was seen in six out of $22(27.2 \%)$ CSVD lesions (Figure 3).

Data are summarised in Table 1.

The difference in the proportion of white matter hyperintense lesions that presented with the central vein sign on SWI images in patients with $\mathrm{MS}$ and CSVD was statistically different $(P<0.5)$. The analysis of the proportion of lesions with the central vein sign at patient levels showed that a significantly higher number of MS patients presented with lesions with the central vein sign compared to CSVD patients, particularly in periventricular distributed comparison (18 MS patients vs. $11 \mathrm{CSVD}$ patients, $P<0.5)$.

\section{Discussion}

In this study, we detected the central vein sign more frequently in patients with MS than in patients with CSVD. Moreover, the periventricular distribution of the central vein sign was more evident for periventricular MS lesions than for periventricular CSVD lesions.

These results reinforce the findings of previous studies, ${ }^{9,11-15}$ and support the potential role of the central

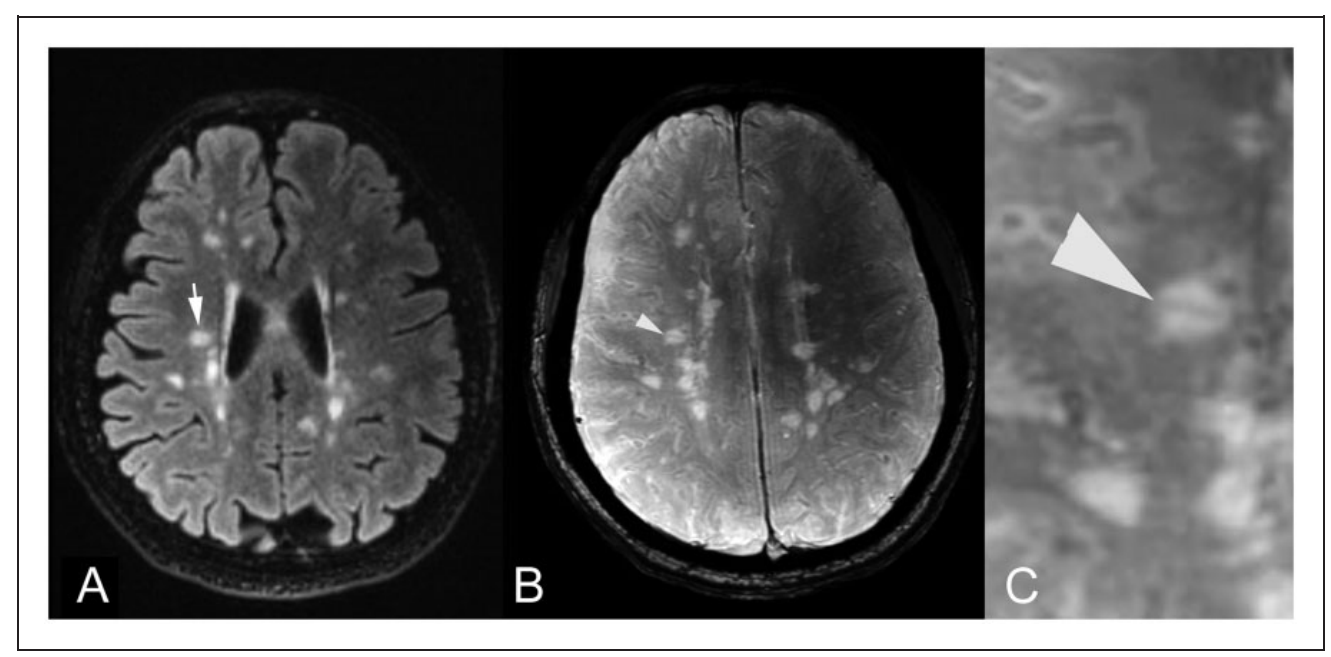

Figure 1. Axial (a) FLAIR MR image shows a T2 hyperintense MS periventricular lesion (arrow). On corresponding (b) SWI image and (c) magnified SWI image, the central vein sign was identified as a linear signal void indicating a small central vein within the MS lesion (arrowhead). FLAIR: fluid-attenuated inversion recovery; MR: magnetic resonance; MS: multiple sclerosis; SWI: susceptibility-weighted imaging. 


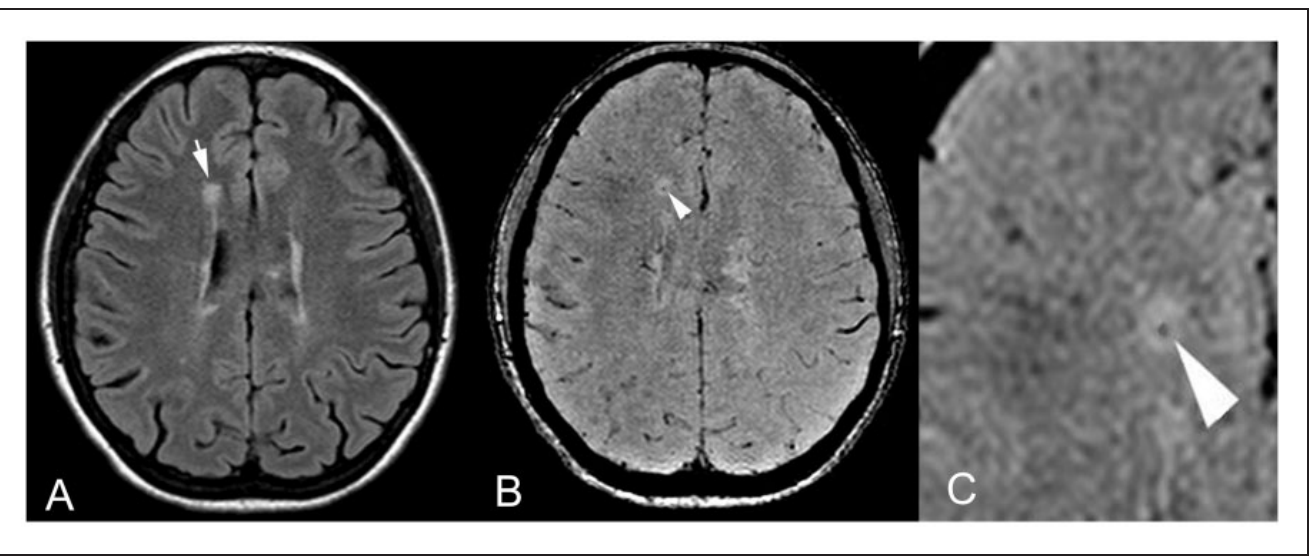

Figure 2. Axial (a) FLAIR MR image shows a T2 hyperintense periventricular MS lesion (arrow). On corresponding (b) SWI image and (c) magnified SWI image, the central vein sign was identified as a central punctate vein showing a signal void within the MS lesion (arrowhead). FLAIR: fluid-attenuated inversion recovery; MR: magnetic resonance; MS: multiple sclerosis; SWI: susceptibility-weighted imaging.

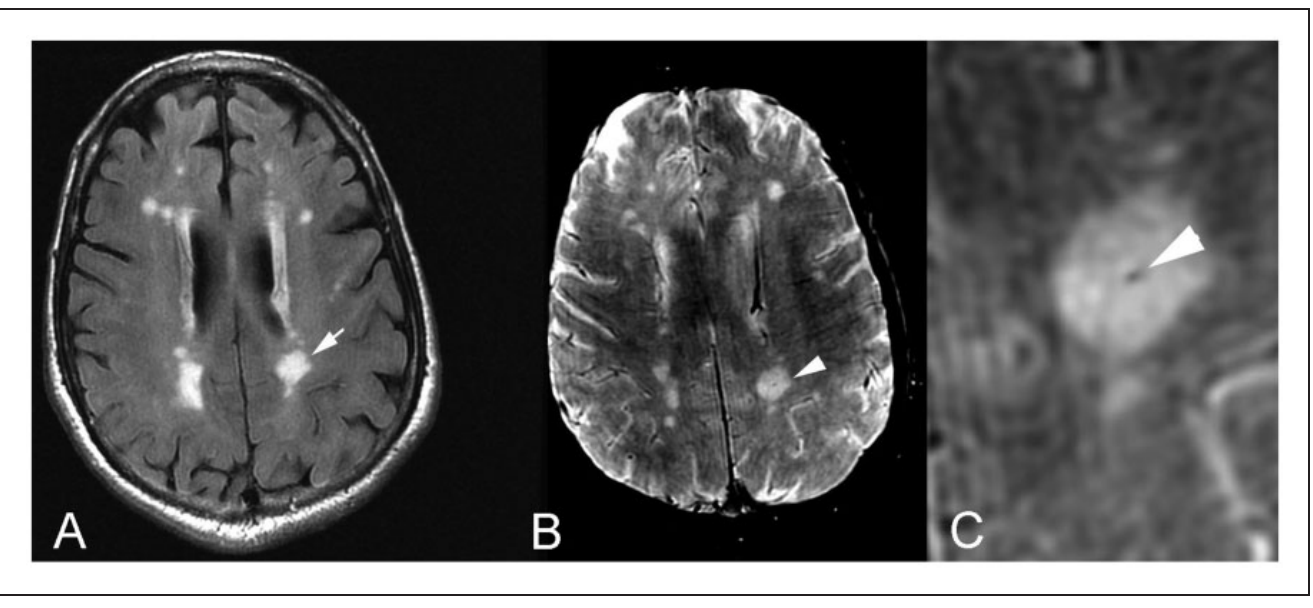

Figure 3. Axial (a) FLAIR MR image shows a T2 hyperintense periventricular CSVD lesion (arrow). On corresponding (b) SWI image and (c) magnified SWI image, the central vein sign was identified as a central punctate vein showing a signal void within the CSVD lesion (arrowhead). FLAIR: fluid-attenuated inversion recovery; MR: magnetic resonance; CSVD: cerebral small vessel disease; SWI: susceptibility-weighted imaging.

vein sign as adjunctive diagnostic criteria for MS as an imaging biomarker of the disease. Small cerebral veins within MS plaques were depicted using a SWI contrast mechanism that exploits tissue magnetic susceptibility differences to generate the contrast. ${ }^{15-19}$ The higher frequency observed in our study of the central vein sign in MS lesions, compared with CSVD lesions, reflects the different mechanism of lesion development.

The observation that most MS lesions showing the central vein sign had a periventricular distribution (Figures 1 and 2) reflect the fact that the process of MS starts around small cerebral veins, as demonstrated by pathology and immunocytochemistry. The perivascular space surrounding these veins is thought to be a privileged site for immune cells to interact with antigenpresenting cells, which can then trigger an inflammatory cascade leading to the formation of lesions around the veins. ${ }^{15,19}$
To better differentiate the central veins from their mimickers, such as microbleeds and calcification, MinIP reformatted SWI images are valuable in demonstrating the continuity of the central veins along their course. $^{20}$ The central vein sign, however, can be found in other neurological diseases such CSVD and migraine. ${ }^{21-23}$ While Lummel et al. ${ }^{24}$ have reported that the central vein sign was equally present in MS and CSVD lesions, multiple recent studies at ultrahigh-field have reported a significantly lower proportion $(45 \%$ at most) of vasocentric white matter lesions in CSVD, thus supporting the hypothesis that the presence of a central vein improves differentiation of MS and vascular lesions. ${ }^{9,10,23}$

As small veins are ubiquitous and may traverse any type of white matter lesion coincidentally, the definition of the central vein sign should be refined by considering target lesions greater than $5 \mathrm{~mm}$ in the largest diameter 
Table 1. Lesion distribution and characteristics presented as number and percentage.

\begin{tabular}{|c|c|c|c|c|}
\hline & $\begin{array}{l}\text { Total no. of } \\
\text { MS lesions }\end{array}$ & $\begin{array}{l}\text { Total no. of } \\
\text { CSVD lesions }\end{array}$ & $\begin{array}{l}\text { No. of MS } \\
\text { lesions with CVS }\end{array}$ & $\begin{array}{l}\text { No. of CSVD } \\
\text { lesions with CVS }\end{array}$ \\
\hline $\begin{array}{l}\text { Total (periventricular and non-periventricular } \\
\text { distributed) }\end{array}$ & 313 & 75 & $128(40.9 \%)^{b}$ & $22(29.3 \%)^{d}$ \\
\hline Periventricular distributed & $139(44.4 \%)^{a}$ & $31(41.3 \%)^{a}$ & $71(55.5 \%)^{c}$ & $6(27.2 \%)^{\mathrm{e}}$ \\
\hline Non-periventricular distributed & $174(55.6 \%)^{a}$ & $44(58.7 \%)^{a}$ & $57(44.5 \%)^{c}$ & $16(72.8 \%)^{\mathrm{e}}$ \\
\hline a) Juxtacortical & 50 & 6 & 16 & 1 \\
\hline b) Subcortical & 38 & 12 & 14 & 8 \\
\hline c) Deep white matter & 66 & 18 & 26 & 6 \\
\hline d) Cerebellar & 20 & 8 & 1 & 1 \\
\hline
\end{tabular}

CVS: central vein sign; CSVD: cerebral small vessel disease; MS: multiple sclerosis. Data are given as number and percentage (\%) relative to: ${ }^{\text {Percentage of }}$ periventricular distributed over non-periventricular distributed lesions.

${ }^{b}$ Percentage of MS lesions with the CVS over the total number of MS lesions.

'Percentage of periventricular distributed MS lesions with the CVS over the non-periventricular distributed MS lesions with the CVS.

${ }^{d}$ Percentage of CSVD lesions with the CVS over the total number of CSVD lesions.

e Percentage of periventricular distributed CSVD lesions with the CVS over the non-periventricular distributed CSVD lesions with the CVS.

and limiting the overall number of white matter lesions greater than $3 \mathrm{~mm}$ to a maximum of eight, which is a critical lesion load regarding the Barkhof criteria for the diagnosis of $\mathrm{MS}^{7}$

Kau et al., ${ }^{7}$ using this refined criteria, reported the very high power of discrimination of the central vein sign on SWI images for differentiation between white matter lesions associated with MS and to non-MS disease. The relatively low percentage of the central vein sign in MS lesions reported in this study could be related to higher field strength or the use of different sequences (e.g. FLAIR* sequences) used in other studies. ${ }^{9-11,22-24}$ Maggi et al. ${ }^{25}$ reported that SWI images obtained after contrast media administration improved the contrast between the hypointense central vein and the surrounding hyperintense MS lesion. This aspect requires further validation in a large series of patient populations and could be considered in our future research.

The main limitation of this study was that the patient population was small, thus our results should be confirmed in large series. Another limitation was that the study design was retrospective; however, we selected all consecutive patients with MS. Finally, there is a significant difference in age between the MS and CSVD patients, which reflects the different age distribution of these two diseases.

\section{Conclusion}

In conclusion, our study, using the SWI sequence without contrast media administration, demonstrated the presence of the central vein sign in $40.9 \%$ of MS lesions, most of them periventricular distributed $(55.5 \%)$, while the central vein sign was found in $29.3 \%$ of CSVD lesions with only $27.2 \%$ of periventricular distributed CSVD lesions.

The difference in the proportion of white matter lesions that presented with the central vein sign on SWI images in patients with MS and CSVD was statistically significant, and a significantly higher number of
MS patients presented with lesions with the central vein sign compared to CSVD patients, particularly in periventricular distributed comparison. This finding improves the understanding of the periventricular distribution of MS lesions and could contribute as adjunctive diagnostic criteria for MS disease.

\section{Conflict of interest}

The authors declared no potential conflicts of interest with respect to the research, authorship, and/or publication of this article.

\section{Funding}

This research received no specific grant from any funding agency in the public, commercial, or not-for-profit sectors.

\section{ORCID iD}

Gianvincenzo Sparacia (D) http://orcid.org/0000-0002-47871634

\section{References}

1. Noseworthy JH, Lucchinetti C, Rodriguez M, et al. Multiple sclerosis. N Engl J Med 2000; 343: 938-952.

2. Polman $\mathrm{CH}$, Reingold SC, Banwell B, et al. Diagnostic criteria for multiple sclerosis: 2010 revisions to the McDonald criteria. Ann Neurol 2011; 69: 292-302.

3. Miller DH, Weinshenker BG, Filippi M, et al. Differential diagnosis of suspected multiple sclerosis: a consensus approach. Mult Scler 2008; 14: 1157-1174.

4. Charil A, Yousry TA, Rovaris M, et al. MRI and the diagnosis of multiple sclerosis: expanding the concept of “no better explanation". Lancet Neurol 2006; 5: 841-852.

5. Filippi M, Rocca MA, Barkhof F, et al. Attendees of the Correlation between Pathological MRI Findings in MS Workshop. Association between pathological and MRI findings in multiple sclerosis. Lancet Neurol 2012; 11: 349-360.

6. Fog T. The topography of plaques in multiple sclerosis with special reference to cerebral plaques. Acta Neurol Scand Suppl 1965; 15: 1-161. 
7. Kau T, Taschwer M, Deutschmann H, et al. The "central vein sign": is there a place for susceptibility weighted imaging in possible multiple sclerosis? Eur Radiol 2013; 23: 1956-1962.

8. Lane JI, Bolster B, Campeau NG, et al. Characterization of multiple sclerosis plaques using susceptibility-weighted imaging at $1.5 \mathrm{~T}$ : can perivenular localization improve specificity of imaging criteria? J Comput Assist Tomogr 2015; 39: 317-320.

9. Kilsdonk ID, Wattjes MP, Lopez-Soriano A, et al. Improved differentiation between MS and vascular brain lesions using FLAIR* at 7 Tesla. Eur Radiol 2014; 24: 841-849.

10. Mistry N, Abdel-Fahim R, Samaraweera A, et al. Imaging central veins in brainlesions with $3-\mathrm{T} \mathrm{T} 2 *_{-}$ weighted magnetic resonance imaging differentiates multiple sclerosis from microangiopathic brain lesions. Mult Scler 2016; 22: 1289-1296.

11. Campion T, Smith RJP, Altmann DR, et al. FLAIR* to visualize veins in white matter lesions: a new tool for the diagnosis of multiple sclerosis? Eur Radiol 2017; 27: 4257-4263.

12. Filippi M, Rocca MA, Ciccarelli O, et al.; MAGNIMS Study Group. MRI criteria for the diagnosis of multiple sclerosis: MAGNIMS consensus guidelines. Lancet Neurol 2016; 15: 292-303.

13. Rovira À, Wattjes MP, Tintoré M, et al.; MAGNIMS study group. Evidence-based guidelines: MAGNIMS consensus guidelines on the use of MRI in multiple sclerosis - clinical implementation in the diagnostic process. Nat Rev Neurol 2015; 11: 471-482.

14. Traboulsee A, Simon JH, Stone L, et al. Revised recommendations of the Consortium of MS Centers task force for a standardized MRI protocol and clinical guidelines for the diagnosis and follow-up of multiple sclerosis. AJNR Am J Neuroradiol 2016; 37: 394-401.

15. Sati P, Oh J, Constable RT, et al.; NAIMS Cooperative. The central vein sign and its clinical evaluation for the diagnosis of multiple sclerosis: a consensus statement from the North American Imaging in Multiple Sclerosis Cooperative. Nat Rev Neurol 2016; 12: 714-722.
16. Reichenbach JR, Venkatesan R, Schillinger DJ, et al. Small vessels in the human brain: MR venography with deoxyhemoglobin as an intrinsic contrast agent. Radiology 1997; 204: 272-277.

17. Sparacia G, Agnello F, La Tona G, et al. Assessment of cerebral microbleeds by susceptibility-weighted imaging in Alzheimer's disease patients: a neuroimaging biomarker of the disease. Neuroradiol J 2017; 30: 330-335.

18. Sparacia G, Speciale C, Banco A, et al. Accuracy of SWI sequences compared to $\mathrm{T} 2 \%$-weighted gradient echo sequences in the detection of cerebral cavernous malformations in the familial form. Neuroradiol $J$ 2016; 29: 326-335.

19. Adams CW, Poston RN and Buk SJ. Pathology, histochemistry and immunocytochemistry of lesions in acute multiple sclerosis. J Neurol Sci 1989; 92: 291-306.

20. Öztoprak B, Öztoprak I and Yıldız ÖK. The effect of venous anatomy on the morphology of multiple sclerosis lesions: a susceptibility-weighted imaging study. Clin Radiol 2016; 71: 418-426.

21. Solomon AJ, Schindler MK, Howard DB, et al. "Central vessel sign" on 3T FLAIR* MRI for the differentiation of multiple sclerosis from migraine. Ann Clin Transl Neurol 2015; 3: 82-87.

22. Lamot U, Avsenik J, Šega S, et al. Presence of central veins and susceptibility weighted imaging for evaluating lesions in multiple sclerosis and leukoaraiosis. Mult Scler Relat Disord 2017; 13: 67-72.

23. Samaraweera APR, Clarke MA, Whitehead A, et al. The central vein sign in multiple sclerosis lesions is present irrespective of the T2* sequence at 3 T. J Neuroimaging 2017; 27: 114-121.

24. Lummel N, Boeckh-Behrens T, Schoepf V, et al. Presence of a central vein within white matter lesions on susceptibility weighted imaging: a specific finding for multiple sclerosis? Neuroradiology 2011; 53: 311-317.

25. Maggi P, Mazzoni LN, Moretti M, et al. SWI enhances vein detection using gadolinium in multiple sclerosis. Acta Radiol Open 2015; 4: 1-4. 